\title{
Electronic and Magnetic Reconstructions in Manganite Superlattices
}

\author{
Kalpataru Pradhan and Arno P. Kampf \\ Center for Electronic Correlations and Magnetism, Theoretical Physics III, \\ Institute of Physics, University of Augsburg, D-86135 Augsburg, Germany
}

(Dated: February 19, 2018)

\begin{abstract}
We investigate the electronic reconstruction at the interface between ferromagnetic metallic (FM) and antiferromagnetic insulating (AFI) manganites in superlattices using a two-orbital doubleexchange model including superexchange interactions, Jahn-Teller lattice distortions, and long range Coulomb interactions. The magnetic and the transport properties critically depend on the thickness of the AFI layers. We focus on superlattices where the constituent parent manganites have the same electron density $n=0.6$. The induced ferromagnetic moment in the AFI layers decreases monotonically with increasing layer width, and the electron-density profile and the magnetic structure in the center of the AFI layer gradually return to the bulk limit. The width of the AFI layers and the charge-transfer profile at the interfaces control the magnitude of the magnetoresistance and the metal-insulator transition of the FM/AFI superlattices.
\end{abstract}

\section{INTRODUCTION}

Correlated electron materials often involve the competition between various ordering tendencies of charge, orbital, spin, and lattice degrees of freedom. If further supplemented by weak disorder cluster coexistence, percolative transport, and colossal response phenomena emerge. It therefore remains a continuing challenge to understand the functional properties of transitionmetal oxides $(\mathrm{TMOs})^{1,2}$. The physics at the surface of TMOs is further enriched by atomic and electronic reconstructions and complicated by the lack of inversion symmetry ${ }^{\underline{3}}$. When surfaces of two TMOs were joined together to form an interface ${ }^{4}$, new phenomena were discovered in the last decade. In some cases the phases at the interface are not even realized in the bulk of either of the TMOs which are joined together ${ }^{3,5}$. For instance the discovery of the two-dimensional electron liquid $\underline{\underline{6}}$ which forms at the interface between the insulators $\mathrm{LaAlO}_{3}$ and $\mathrm{SrTiO}_{3}$ started a new subfield in the research on oxide interfaces. Subsequent experiments revealed superconductivity $\underline{\underline{7}}$, ferromagnetism ${ }^{\underline{8}}$, and even their coexistence $e^{\underline{\underline{ }}}$. These unexpected phases at interfaces pose fundamental physics questions and simultaneously bear promises of technological importance for the design of novel materials 10 .

The perovskite manganese oxides are a particularly remarkable example for the mutual coupling of electronic and lattice degrees of freedom $11-13$. The manganites $\mathrm{RE}_{1-x} \mathrm{AE}_{x} \mathrm{MnO}_{3}$, where $\mathrm{RE}$ and $\mathrm{AE}$ denote rare and alkaline earth elements, respectively, are known for their colossal magnetoresistance ${ }^{14}$. The various phases in manganites with charge, orbital, and magnetic order have been elaborated for different combination of RE and $\mathrm{AE}$ elements and doping regimes $x^{2,11,15,16}$. The recent development in superlattices has created yet another tool to explore electronic and magnetic phases of manganites.

$\mathrm{La}_{1-x} \mathrm{Sr}_{x} \mathrm{MnO}_{3}$, a solid solution of $\mathrm{LaMnO}_{3}$ (LMO) and $\mathrm{SrMnO}_{3}$ (SMO) exhibits a number of phases depending upon the doping concentration $x$. In addition to A-type at $x=0$ and $\mathrm{G}$-type antiferromagnetism at $x=1 \frac{17}{}$ also ferromagnetic and C-type antiferromagnetic (AF) phases exist at low temperature in an intermediate doping regime. For example $\mathrm{La}_{0.67} \mathrm{Sr}_{0.33} \mathrm{MnO}_{3}$ is a ferromagnetic metal and $\mathrm{La}_{0.33} \mathrm{Sr}_{0.67} \mathrm{MnO}_{3}$ is an $\mathrm{AF}$ insulator $16.18,19$. In manganite superlattices $2 \mathrm{~m}$ layers of LMO are deposited on $\mathrm{m}$ layers of $\mathrm{SMO}^{20,21}$ or vice versa 22 . For $m=1, \mathrm{LMO}_{2 m} / \mathrm{SMO}_{m}$ and $\mathrm{LMO}_{m} / \mathrm{SMO}_{2 m}$ are the superlattice counterpart of the $\mathrm{La}_{0.67} \mathrm{Sr}_{0.33} \mathrm{MnO}_{3}$ and $\mathrm{La}_{0.33} \mathrm{Sr}_{0.67} \mathrm{MnO}_{3}$ manganites, respectively.

For $\mathrm{LMO}_{2 m} / \mathrm{SMO}_{m}$ superlattices the uniform FM behavior of the solid solution is recovered for $m \leq 2$. A metal-insulator transition (MIT) occurs for $m \geq 3$ accompanied by a strongly modulated magnetic structure across the interface 20,21 . Similarly, $\mathrm{LMO}_{m} / \mathrm{SMO}_{2 m}$ shows a C-type AF phase for $m=1$ and $m=2$ that exist in the solid solution counterpart $\mathrm{La}_{0.33} \mathrm{Sr}_{0.67} \mathrm{MnO}_{3}$. The Néel temperature $250 \mathrm{~K}$ of the solid solutions increases to $320 \mathrm{~K}$ for $m=1$. For $m \leq 2$, charge transfer from LMO to SMO may retain properties similar to the solid solutions. In these two scenarios, the charge transfer is likely confined to 1-3 unit cells. For $m>2$, the increasing distance between the interfaces limits the charge transfer to the near vicinity of the interface. Due to this charge confinement LMO and SMO regain their respective parent phases away from the interface.

Considerable progress has been achieved in describing the modulated magnetic structure $\underline{23}-27$ and the MIT 27 in the LMO/SMO superlattices. The standard two-orbital double exchange model for manganites was implemented to calculate the average electron density for each layer of the LMO/SMO superlattices; away from the interface LMO and SMO layers regain their initial electron densities. The electron-density profile across the interface is determined by electrostatics with an electron transfer from the LMO to the SMO side. The magnetic-profile for each layer follows the bulk phase diagram at the corresponding local density along with a weak proximity effect 23,27 .

In the initial studies of manganite superlattices $28-31$ the primary intent was to improve the tunneling 
magnetoresistance $\frac{32,33}{2}$ in a FM/AFI superlattice where the constituent parent FM and AFI manganites have the same electron density, $n=1-x$. FM/AFI superlattices with different combinations of manganites (FM = LSMO, LCMO; AFI = PCMO, GdCMO; $n=0.67)+30,34-36$ have been studied in recent years. The observed induced ferromagnetic moment in the AFI layers depends on their width and the induced moment is tunable by an external magnetic field $30,36,37$. Remarkably the required external field is much smaller than the magnetic field required to induce ferromagnetism in the parent AFI compound. This results in a large magnetoresistance in the FM/AFI superlattices which are therefore candidate materials for colossal magnetoresistance at room temperature. Manganite trilayers can also be used to design an efficient spin valve 38 .

In this paper, we have studied in detail the electronic and magnetic reconstructions in FM/AFI superlattices at electron density $n=0.6$ for different widths of the AFI layers. Electrons are transferred from the the FM to the AFI layers at the interface even though the initial electron density in the bulk FM and bulk AFI are equal. Our study confirms the underlying one-to-one correspondence between the density profile and the magnetic profile in the superlattices. With increasing AFI layer width, the induced ferromagnetic moment in the AFI layers decreases monotonically and drives an MIT in the superlattices. In addition to the large magnetoresistance our calculations explain the observed temperature crossover between positive and negative magnetoresistance and the two ferromagnetic transitions observed in the superlattices 30,36 .

The paper is organized as follows: In Sec. II, we introduce the two-orbital model for bulk perovskite manganites. For superlattices we specify the essential modification by adding long-range Coulomb (LRC) interactions and briefly present the applied Monte Carlo technique. The parameter space of the FM/AFI manganite superlattices is discussed in Sec. III. Electronic and magnetic reconstructions at manganite interfaces are emphasized in Sec. IV while Sec. V is devoted to the MIT. Results with and without LRC interactions are compared in Sec. VI. In Sec. VII, various combinations of electron-phonon couplings for FM and AFI manganites are considered. The role of disorder at the interface is discussed in Sec. VIII, and conclusions are presented in Sec. IX.

\section{REFERENCE MODEL FOR MANGANITES}

We construct a two-dimensional model Hamiltonian for manganite superlattices composed of FM regions separated by AFI regions as

$$
H=H_{F M}+H_{A F I}+H_{l r c}
$$

where both $H_{F M}$ and $H_{A F I}$ have the same reference Hamiltonian $39-42$

$$
\begin{aligned}
H_{r e f}= & \sum_{\langle i j\rangle \sigma}^{\alpha \beta} t_{\alpha \beta}^{i j} c_{i \alpha \sigma}^{\dagger} c_{j \beta \sigma}-J_{H} \sum_{i} \mathbf{S}_{i} \cdot \boldsymbol{\sigma}_{i}+J \sum_{\langle i j\rangle} \mathbf{S}_{i} \cdot \mathbf{S}_{j} \\
& -\lambda \sum_{i} \mathbf{Q}_{i} \cdot \boldsymbol{\tau}_{i}+\frac{K}{2} \sum_{i} \mathbf{Q}_{i}^{2}-\mu \sum_{i \alpha \sigma} c_{i \alpha \sigma}^{\dagger} c_{i \alpha \sigma} \cdot(2)
\end{aligned}
$$

Here, $c$ and $c^{\dagger}$ are annihilation and creation operators for itinerant $e_{g}$ electrons and $\alpha, \beta$ refer to the two Mn$e_{g}$ orbitals $d_{x^{2}-y^{2}}$ and $d_{3 z^{2}-r^{2}}$ labelled as $a$ and $b$, respectively. The kinetic energy part involves the nearestneighbor hopping of $e_{g}$ electrons with amplitude $t_{\alpha \beta}^{i j}$ $\left(t_{a a}^{x}=t_{a a}^{y} \equiv t, t_{b b}^{x}=t_{b b}^{y} \equiv t / 3, t_{a b}^{x}=t_{b a}^{x} \equiv-t / \sqrt{3}, t_{a b}^{y}=\right.$ $\left.t_{b a}^{y} \equiv t / \sqrt{3}\right)^{40}$, where $x$ and $y$ denote the spatial directions on a square lattice. $J_{H}$ is the Hund's rule coupling between $t_{2 g}$ spins $\mathbf{S}_{i}$ and the $e_{g}$ electron spin $\boldsymbol{\sigma}_{i}$, and $J$ is the AF superexchange between the $t_{2 g}$ spins. $\lambda$ measures the strength of the electron-phonon coupling between the Jahn-Teller (JT) distortions, $Q_{2 i}, Q_{3 i}$ and the orbital pseudo spin operators $\tau_{i 2}=\sum_{\sigma}\left(c_{i a \sigma}^{\dagger} c_{i b \sigma}+c_{i b \sigma}^{\dagger} c_{i a \sigma}\right)$, $\tau_{i 3}=\sum_{\sigma}\left(c_{i a \sigma}^{\dagger} c_{i a \sigma}-c_{i b \sigma}^{\dagger} c_{i b \sigma}\right)$. Here $Q_{2 i}$ and $Q_{3 i}$ are the distortions corresponding to the normal vibration modes of the oxygen octahedron that remove the degeneracy of the $\mathrm{e}_{g}$ levels. The stiffness of the JT modes is denoted by $K$. The stiffness of the breathing mode distortion $\left(Q_{1 i}\right)$, which couples to the local electron density is considerably larger than $K$ and therefore, in the adiabatic approximation the coupling to $Q_{1 i}$ is neglected ${ }^{40}$ in the Hamiltonian Eq. 2.

We treat all $t_{2 g}$ spins and lattice degrees of freedom as classical ${ }^{43,44}$, and measure energies in units of the MnMn hopping $t_{a a}=t$. In manganites $t$ is approximately 0.2-0.5 $\mathrm{eV}^{11,45}$. The estimated value of the Hund's coupling is $2 \mathrm{eV}^{46}$, i.e. much larger than $t$. For this reason we further adopt the limit $J_{H} \rightarrow \infty$, for which the $e_{g}$ electron spin perfectly aligns along the local $t_{2 g}$ spin direction. The infinite Hund's rule coupling then naturally leads to redefine the spinless $e_{g}$ electron operator as $c_{i \alpha}=\cos \left(\theta_{i} / 2\right) c_{i \alpha \uparrow}+\sin \left(\theta_{i} / 2\right) e^{-i \phi_{i}} c_{i \alpha \downarrow}$, where the polar angle $\theta_{i}$ and the azimuthal angle $\phi_{i}$ determine the $t_{2 g}$ spin orientation. In terms of the redefined $\mathrm{e}_{g}$ electron operators, the kinetic energy takes the simpler form

$$
H_{\mathrm{kin}}=-\sum_{<i j>, \alpha \beta} \tilde{t}_{\alpha \beta}^{i j} c_{i \alpha}^{\dagger} c_{j \beta},
$$

where $\tilde{t}_{\alpha \beta}^{i j}$ is defined as $\tilde{t}_{\alpha \beta}^{i j}=\Theta_{i j} t_{\alpha \beta}^{i j}$ with $\Theta_{i j}=$ $\cos \left(\theta_{i} / 2\right) \cos \left(\theta_{j} / 2\right)+\sin \left(\theta_{i} / 2\right) \sin \left(\theta_{j} / 2\right) e^{-i\left(\phi_{i}-\phi_{j}\right)}$. The factor controls the magnitude of the hopping amplitudes due to the different orientation of $t_{2 g}$ spins at sites $i$ and $j$.

We set $K=1$ without loss of generality, because if the lattice variable $\mathrm{Q}$ is replaced by $\sqrt{K} Q, \lambda$ can be simply renormalized as $\lambda / \sqrt{K}$. The length of the $t_{2 g}$ spins is set to $\left|\mathbf{S}_{i}\right|=1$. In an external magnetic field a Zeeman coupling $H_{m a g}=-\mathbf{h} \cdot \sum_{i} \mathbf{S}_{i}$ is added to the Hamiltonian. 
The reference 'manganite model' $H_{\text {ref }}$ in $2 \mathrm{D}$ is constructed to reproduce the correct sequence of magnetic phases in the bulk limit 22.47 . The different phases that are captured at low temperature are an orbitally ordered insulator at $x=0 \underline{41}$, a charge and orbital ordered ferromagnetic insulator at $x=0.25^{\underline{47}}$, a ferromagnetic metal window around $x=0.67$, the CE charge and orbital ordered insulator around $x=0.50$, a magnetic two dimensional A-type AF phase, and a G-type AF phase at $x=$ 1.00 .

The average electron density of the constituent FM and AFI manganites in the FM/AFI superlattices is fixed by choosing the same chemical potential $\mu$ throughout the superlattice. The LRC interaction between all the charges, essential to control the amount of charge transferred across the interface is taken into account via a self consistent solution of the Coulomb potentials $\phi_{i}$ at the mean-field level by setting $\underline{48}$

$$
\phi_{i}=\alpha t \sum_{j \neq i} \frac{\left\langle n_{j}\right\rangle-Z_{j}}{\left|\mathbf{R}_{i}-\mathbf{R}_{j}\right|}
$$

in the long-range Coulomb part of the Hamiltonian,

$$
H_{l r c}=\sum_{i} \phi_{i} n_{i}
$$

It is assumed that all the point charges $Z_{j}$ from the background ions are fixed and confined to the Mn sites. $\left\langle n_{j}\right\rangle$ refers to the $e_{g}$ electron density at the Mn site $\mathbf{R}_{j}$. The long-range interactions between the background point charges and the $e_{g}$ electrons are thereby combined with the charge-neutrality condition in the superlattice. Alternatively the Coulomb potentials $\phi_{i}$ can be determined self consistently by solving the Poisson equation $\underline{49}$ which is equivalent to solving the Eq. 3 .

The Coulomb interaction strength is controlled by the parameter $\alpha=e^{2} / \epsilon a t$ where $\epsilon$ and $a$ are the dielectric constant and the lattice parameter, respectively. This mean-field level set up is the minimal basis to study the charge transfer across the interface $\frac{23,25,26}{2}$; it is also commonly used in the context of semiconductor interfaces $\underline{50}$.

The background dielectric constant $\epsilon$ is order of 20 in manganites 51,52 . It includes the lattice and the atomic polarizability contributions only. The dipolar contribution from the charge carrier motion and the associated screening is neglected to treat the absolute permittivities of FM and AFI manganites on equal footing 52 . For a lattice constant $a=4 \AA, t=0.5 \mathrm{eV}$, and $\epsilon=20$, the approximate value of $\alpha$ is 0.2 . The screening length in two dimensions (2D) is larger than in three dimensions (3D) ${ }^{53}$. This is taken into account by choosing a larger dielectric constant in the 2D model ansatz. Specifically we use $\alpha=0.1$ in our calculations. The $\alpha$ values employed earlier in $3 \mathrm{D}$ model calculations were in the range $0.1-123,25,26$ while a much smaller value of $\alpha$ was used in $1 \mathrm{D}^{54}$.
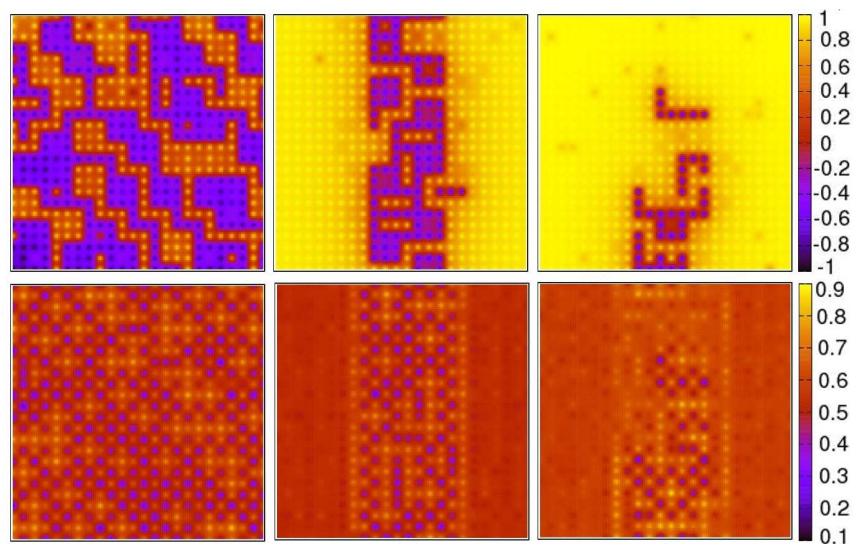

(a)

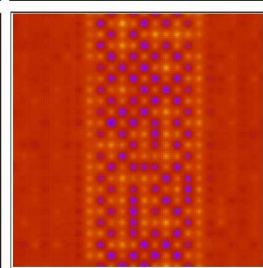

(b)

(c)

FIG. 1: Color online: 1st row: The $\mathrm{z}$ components of the $t_{2 g}$ spins; 2nd row: the electron-density for each site on a $24 \times 24$ lattice at $\mathrm{T}=0.01$. (a) The parent AF insulator, (b) the superlattice with the width $\mathrm{w}=11$ of the AFI layer, (c) the superlattice with $\mathrm{w}=11$ in the presence of an external magnetic field, $\mathrm{h}=0.004$.

We apply an exact diagonalization scheme to the itinerant electron system for each configuration of the background classical variables of the $t_{2 g}$ spins and the lattice distortions. The classical variables are annealed by starting from a random configuration. At each temperature the annealing requires at least 2000 system sweeps, by visiting every site of the lattice sequentially and updating the system by a metropolis algorithm. For each system sweep the computation time scales as $\mathrm{O}\left(N^{4}\right)$ where $\mathrm{N}$ is the number of lattice sites. With thousands of annealing sweeps at various temperatures this procedure is time consuming.

We instead resort to a Monte Carlo sampling technique based on the 'traveling cluster approximation' (TCA) ${ }^{55}$. The TCA uses a moving cluster of size $N_{c}$ constructed around the site of the Monte Carlo update. The computational cost thereby decreases to $\mathrm{O}\left(N N_{c}^{3}\right)$ and allows to treat system sizes up to $\sim 40 \times 40$, with an $8 \times 8$ moving cluster. The method and the associated transport calculations for $\mathrm{N}=24 \times 24$ were extensively benchmarked and successfully applied in several earlier studies $41,42,47$. At each system sweep we additionally solve for the Coulomb potentials $\phi_{i}$ in $H_{l r c}$ self consistently until the electron density at each site is converged. The maximum relative error for the convergence of the electron density is set to 0.01 at low temperatures while it is relaxed up to 0.03 at higher temperatures. For each fixed set of parameters, the calculations start from ten different initial realizations of the classical variables. All the physical quantities are averaged over the results for these ten starting configurations. 

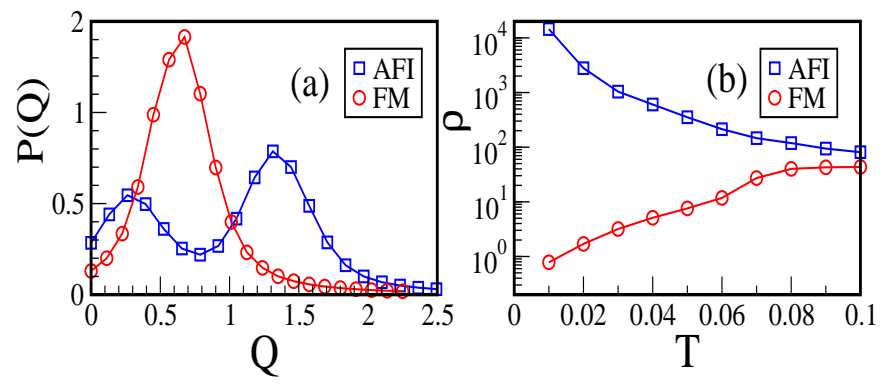

FIG. 2: Color online: Bulk system at $n=0.6$ (a) Distribution function for the lattice distortions $\mathrm{P}(\mathrm{Q})$ of the bulk AFI $\left(\lambda_{I}=1.75\right)$ and bulk FM $\left(\lambda_{M}=1.50\right)$ at $\mathrm{T}=0.01$. (b) The resistivity variation with temperature for AFI and FM bulk systems.

\section{SUPERLATTICES OF FERROMAGNETIC METALS AND ANTIFERROMAGNETIC INSULATORS}

Here we analyze specifically superlattices composed of FM and AFI manganites. Even with the above described simplifications in the model Hamiltonian we have to contend with manganite states at different doping $x$, AF superexchange strengths $J$, and different electron-phonon couplings $\lambda$. Also the proper choice of relevant parameters for the combination of ferromagnetic metals and $\mathrm{AF}$ insulators is not obvious. In addition, due to the slight structural and chemical mismatch between the parent FM and AFI manganites the parameters at the interface may be altered with respect to the bulk values.

To constrain the parameter space we select the FM and AFI manganites of equal electron density $n=1-x$. We choose $n=0.6$ above half filling to address the existing experiments on manganite superlattices near $x=1 / 3$. The AF superexchange couplings may vary depending on the different combinations of $\mathrm{RE}$ and $\mathrm{AE}$ ions. But for simplicity we will use the typical value $J=0.1^{56}$ for both the FM and the AFI manganites. With the choice of the electron density and the superexchange couplings we are left with the crucial parameter $\lambda$ to differentiate between a FM and an AFI phase.

Alternatively $\lambda$ can be fixed and the differentiation between the ferromagnetic metal and the AF insulator is achieved by varying $J$. This requires a larger value of $J$ in the AF insulator, but it leaves the ambiguity for choosing modified superexchange couplings at the interface. Yet another possibility is to keep each parameter $J$ and $\lambda$ fixed and to use different hopping amplitudes on the two sides of the interface. Again we are left with the possible modifications for the hopping amplitude at and across the interface. Since the $\lambda$ and $J$ values are measured in units of $t$, a smaller $t$ value is equivalent to the combinations of larger $\lambda$ and $J$ values or vice versa. Smaller and larger $\lambda$ values are thereby closely related to the larger and smaller bandwidth manganites, respectively.
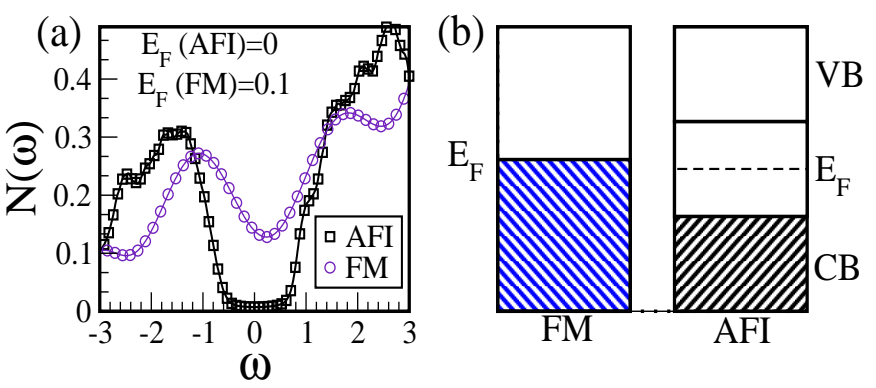

FIG. 3: Color online: Bulk system at $n=0.6$ (a) DOS of the bulk AFI $\left(\lambda_{I}=1.75\right)$ and FM $\left(\lambda_{M}=1.50\right)$ at $\mathrm{T}=0.01$. (b) Schematic energy diagram.

Here we consider two $\lambda$ values to differentiate between the FM and the AFI manganites. For the parameters $J=0.1$ and the density $n=0.6$, the groundstate is FM for $\lambda \equiv \lambda_{M}=1.50$ while it is an AFI for $\lambda \equiv \lambda_{I}=1.75$. The AFI phase at $n=0.6$ is not a perfect charge and orbital ordered $\mathrm{CE}$ phase which is stable only at $n=$ 0.50 .42 . The AFI phase at $n=0.6$ is inhomogeneous, it contains the CE-type zigzag ferromagnetic chains and local charge ordered regions as shown in Fig.1(a).

We compute the 'one point' distribution function of the lattice distortions, $P(Q)=\left\langle\frac{1}{N} \sum_{i} \delta\left(Q-\left|Q_{i}\right|\right)\right\rangle$ where $\mathrm{N}$ is the total number of sites, to compare them with the electron-density variations. The two peaks in Fig.2(a) for the AFI at $\mathrm{T}=0.01$ indicate that the distortions have a bimodal distribution. The sites with a large distortion attract more electrons with a density increase to $\mathrm{n}_{i} \sim$ 0.8. So the spatial density pattern in Fig.1(a) reflects simultaneously the large and small structural distortions. The single peak structure of $\mathrm{P}(\mathrm{Q})$ for the FM indicates instead that there are distortions of similar strength on all sites, which facilitates the motion of the electrons and thereby supports the metallic character.

We obtain the resistivity for both FM and AFI bulk phases by calculating the d.c. limit of the longitudinal conductivity as determined by the Kubo-Greenwood formula 57

$$
\sigma(\omega)=\frac{A}{N} \sum_{\alpha, \beta}\left(n_{\alpha}-n_{\beta}\right) \frac{\left|f_{\alpha \beta}\right|^{2}}{\epsilon_{\beta}-\epsilon_{\alpha}} \delta\left(\omega-\left(\epsilon_{\beta}-\epsilon_{\alpha}\right)\right),
$$

with $A=\pi e^{2} / \hbar a$ and $n_{\alpha}=\theta\left(\mu-\epsilon_{\alpha}\right), a$ is the lattice spacing. $f_{\alpha \beta}$ denotes the matrix elements of the paramagnetic current operator $j_{x}=i t \sum_{i, \sigma}\left(c_{i+x, \sigma}^{\dagger} c_{i, \sigma}-h . c\right.$. $)$ between eigenstates $\left|\psi_{\alpha}\right\rangle,\left|\psi_{\beta}\right\rangle$, and $\epsilon_{\alpha}, \epsilon_{\beta}$ are the corresponding eigenenergies. We extract the d.c. conductivity by calculating the 'average' conductivity for a small low frequency interval $\Delta \omega$ defined as

$$
\sigma_{a v}(\Delta \omega)=\frac{1}{\Delta \omega} \int_{0}^{\Delta \omega} \sigma(\omega) \mathrm{d} \omega .
$$

$\Delta \omega$ is chosen two to three times larger than the mean finite-size gap of the system as determined by the ratio of the bandwidth and the total number of eigenvalues. This procedure has been benchmarked in a previous 


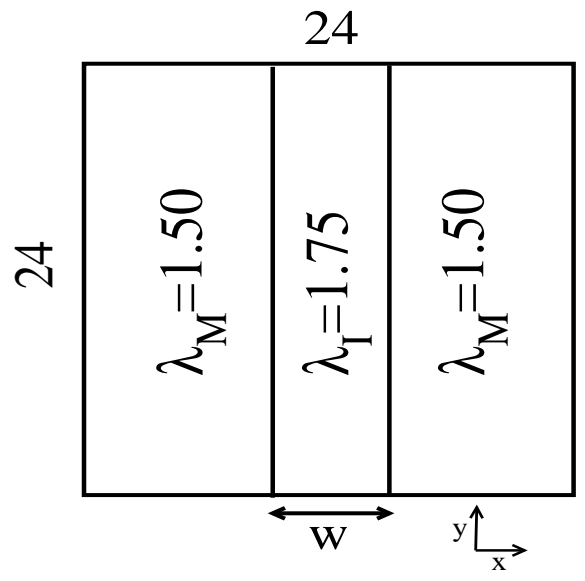

FIG. 4: Schematic view of the FM/AFI superlattice on a $24 \times 24$ lattice. We consider periodic boundary conditions in both directions.

work ${ }^{58}$. Fig.2(b) shows the temperature dependence of the resistivity $\rho=\sigma_{a v}^{-1}$ in units of $\hbar a / \pi e^{2}$. The insulating behavior in the AFI at low temperatures is due to charge and orbital order, which opens an energy gap in the spectrum.

The density of states (DOS) $N(\omega)=\left\langle\frac{1}{N} \sum_{\alpha} \delta\left(\omega-\epsilon_{\alpha}\right)\right\rangle$ is shown in Fig.3(a). The center of the gap in the DOS of the AFI state is chosen as the energy zero. With this choice the Fermi energy of the FM state at the same density is at $\epsilon_{F}=0.1$. The DOS is finite for the FM state at its Fermi level. Fig.3(b) translates the results for the DOS into a schematic energy diagram. When the FM and the AFI manganites are joined together band bending near the interface is expected to shift electrons from the FM to the AFI side. An FM/AFI superlattice is shown schematically in Fig.4. The width of the middle AFI layer, sandwiched between the FM layers is denoted by w. Periodic boundary conditions are enforced in both directions and thereby represent a superlattice structure composed of alternating FM and AFI regions.

\section{CHARGE TRANSFER AND MAGNETIC RECONSTRUCTION AT THE INTERFACE}

As we show below, if the width of the AF regions is small, they may become ferromagnetic and metallic in the superlattice structure. The induced magnetization is restricted to the near vicinity of the interfaces. The distance from the interface at which the parent $\mathrm{AF}$ insulating character is recovered depends upon the parameter $\alpha$ and the electron-phonon couplings $\lambda_{M}$ and $\lambda_{I}$.

In this section we start with the specific choice of $\lambda_{M}=1.50, \lambda_{I}=1.75$, and $\alpha=0.1$, and discuss results for the decrease of the magnetization with increasing width of the AFI layers. This decrease is quantified by the average z component of the $t_{2 g}$ spins $\left\langle S_{z}\right\rangle_{I}$ in the AFI layer, where the angular bracket denotes the ther-
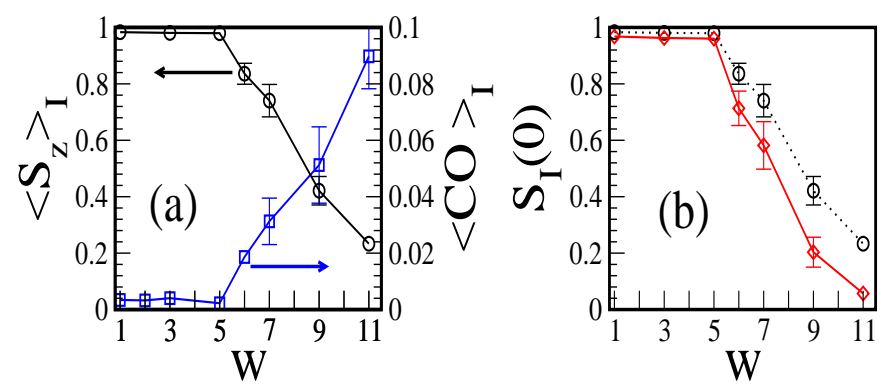

FIG. 5: Color online: The average $\mathrm{z}$ component of $\mathrm{t}_{2 g}$ spins, $\left\langle S_{z}\right\rangle_{I}$ and the average staggered charge order $\langle C O\rangle_{I}$ (see text) in the AFI layer at $\mathrm{T}=0.01$. (b) Ferromagnetic structure factor $\mathrm{S}_{I}(\mathbf{0})$ for different widths of the AFI layer at $\mathrm{T}=0.01$. The average $\mathrm{z}$ component is also re-plotted as the dotted line.

mal average combined with an additional average over ten different 'samples'. We also define a measure for the local staggered charge order by $\langle C O\rangle_{I}=\frac{1}{N_{I}} \sum_{i \in A F I}\left\langle n_{i}\right\rangle$ $\mathrm{e}^{i(\pi, \pi) \cdot \mathbf{r}_{i}}$ for the AFI layer; $i$ denotes one of the $N_{I}$ lattice sites in the AFI layer with position $\mathbf{r}_{i}$. In Fig.5(a), we plot $\left\langle S_{z}\right\rangle_{I}$ and $\langle C O\rangle_{I}$ for different widths w. For $\mathrm{w} \leq 5$, the averaged $\left\langle S_{z}\right\rangle_{I}$ is near unity and starts to decrease for $\mathrm{w}>5$, while $\langle C O\rangle_{I}$ remains small for $\mathrm{w} \leq 5$ and starts to rise for $\mathrm{w}>5$. Similarly (see Fig.5(b)) also the long-wavelength magnetic structure factor $S_{I}(\mathbf{0})$ starts to decrease beyond $\mathrm{w}=5$, where $S_{I}(\mathbf{q})=\frac{1}{N_{I}^{2}} \sum_{i j \in A F I}$ $\left\langle\mathbf{S}_{\mathbf{i}} \cdot \mathbf{S}_{\mathbf{j}}\right\rangle \mathrm{e}^{i \mathbf{q} \cdot\left(\mathbf{r}_{i}-\mathbf{r}_{j}\right)}$. So the decrease in the magnetization beyond a critical width $\mathrm{w}_{c}$ is accompanied by emerging charge order in the AFI layer. The critical width for fixed $\lambda_{I}$ may change for a different set of parameters $\lambda_{M}$ and $\alpha$. This will be discussed in sections VI and VII. The critical width is therefore not directly related to an intrinsic length scale of the parent bulk AFI.

The averaged magnetization necessarily does not reveal any spatial variation transverse to the interface. In order to analyze the magnetization profile, we calculate the $\left\langle S_{z}(x)\right\rangle$ for each line of the superlattice with transverse coordinate $x$. Fig.6(a) shows the line averaged $\left\langle S_{z}(x)\right\rangle$ vs. line index for $\mathrm{w}=7 .\left\langle S_{z}(x)\right\rangle$ decreases for $x=12,13$, and 14 , i.e. in the center lines of the AFI layer. So the induced ferromagnetic moment in the AFI layer is indeed confined to just the two lines nearest to the interface.

In the bulk for $\lambda_{I}=1.75$ the AFI phase extends to electron densities larger than $n=0.6$ and changes to a ferromagnetic insulating phase at $n=0.75 \underline{47}$. It is therefore natural to explore the connection between the induced magnetization in the AFI layer with the electron densities at the interface. As shown in Fig. 6(b), at the interface the line averaged electron density $\langle n(x)\rangle$ of the FM layer decreases while $\langle n(x)\rangle$ for the AFI layer increases beyond the initial electron density 0.6. In fact $\langle n(x)\rangle$ at the fully magnetized lines $x=10$ and 16 is nearly equal to 0.75 .

The direction of electron transfer from the FM to the AFI regions was anticipated already in the discussion in 

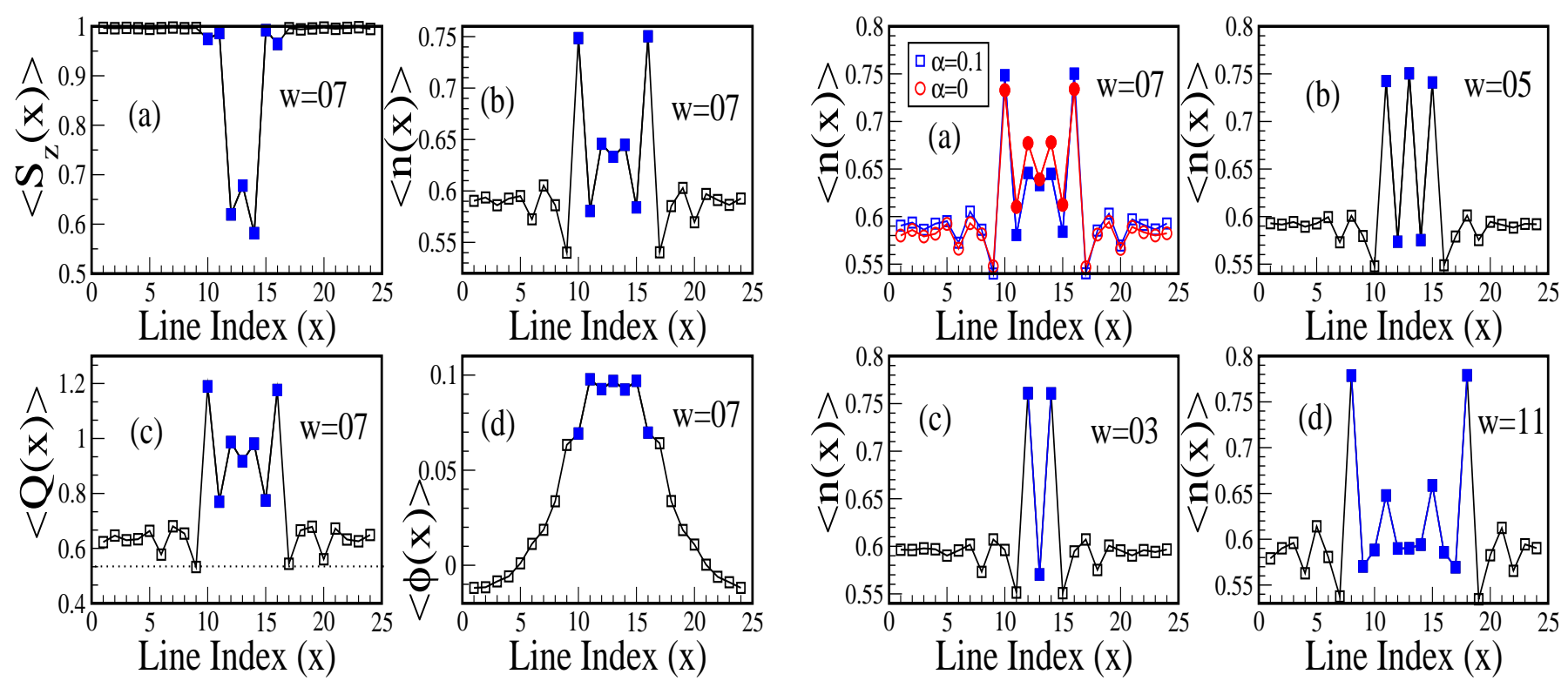

FIG. 6: Color online: Line averaged (a) z component of the $t_{2 g}$ spins $\left\langle S_{z}(x)\right\rangle$, (b) electron density $\langle n(x)\rangle$, (c) LRC potentials $\langle\phi(x)\rangle$, and (d) lattice distortions $\langle Q(x)\rangle$ vs. line index for $\mathrm{w}$ $=7$. Open and closed symbols are for the FM and the AFI layers, respectively. The temperature is $\mathrm{T}=0.01$

Section III. Away from the interface the average electron density gradually returns to the initial electron density $n=0.6$. In addition to the overall charge transfer, $\langle n(x)\rangle$ is spatially modulated perpendicular to the interface. These charge modulations are Friedel-like density oscillations; their amplitude decreases with the distance from the interface. The spatial density variations are nearly symmetric around the central line, $x=13$. As we have verified a perfect symmetry can be achieved by averaging over a larger number of samples.

For the incommensurate filling $n=0.6$, the parent AFI is fragmented into small regions with and without charge and orbital order. High-density sites (see Fig.1(a)) are always accompanied by large lattice distortions. Similarly a one-to-one correspondence between the the electron densities and the lattice distortions exists in the superlattice. The line averaged lattice distortions $\langle Q(x)\rangle$ (see Fig.6(c)) are infact larger in the AFI layer. $\langle Q(x)\rangle$ is modulated similarly to the line averaged electron density shown in Fig.6(b).

The amount of charge transfer from the FM to the AFI layers is controlled by the LRC interactions. The line averaged Hartree potential $\langle\phi(x)\rangle$ in Fig.6(d) is negative for $x \leq 4$ and $x \geq 22$. The average electron densities for those lines is nearly equal to the initial electron density $n=0.6$. The charge transfer is restricted to a few lines near the interface where the averaged potentials $\langle\phi(x)\rangle$ are positive and thereby counteracts further electron transfer. Since $\phi_{i}$ and $n_{i}$ are the self-consistent solutions of the Poisson equation, the increase or the decrease of the averaged electronic density has a one-to-one

FIG. 7: Color online: Line averaged electron density $\langle n(x)\rangle$ for (a) $\mathrm{w}=7$ with $(\alpha=0.1)$ and without $(\alpha=0)$ LRC interaction, (b) $\mathrm{w}=5,(\mathrm{c}) \mathrm{w}=3,(\mathrm{~d}) \mathrm{w}=11$. Open and closed symbols are for the FM and the AFI layers, respectively. The temperature is $\mathrm{T}=0.01$

correspondence to the negative or positive curvature of $\langle\phi(x)\rangle$.

For $\alpha=0.1$, see Fig.7(a), the electron densities are clearly lower (higher) in the AFI (FM) layer as compared to $\alpha=0$. The LRC potentials decrease the electron density in the center of the AFI layer, but the average electron densities for individual lines at $x=12, x=13$, and $x=14$ are still enhanced due to the Friedel-like density oscillations. The electron density in the central line $x=13$ results from the superposition of the oscillations originating from $x=10$ and $x=16$. The constructive superposition is more pronounced for the AFI layer width $\mathrm{w}=5$ shown in Fig.7(b), for which the center line is fully magnetized due to the enhanced electron density and the induced magnetism originating from the neighboring magnetized $2+2$ interfacial lines. For $\mathrm{w}=3$, in Fig.7(c), the electron density of the central line is nearly equal to the initial electron density and the charge transfer is truly confined to the interface. For $\mathrm{w}>7$, density oscillations in the AFI layer disappear due the recovery of the bulk AFI character (see Fig.7(d) for $\mathrm{w}=9$ ).

\section{METAL-INSULATOR TRANSITION}

In this section we compare the magnetic structure factor $S_{I}(\mathbf{0})$ in the AFI layer with the resistivity for the superlattice for current flow perpendicular to the interface. Fig.8(a) shows the temperature dependence of $S_{I}(\mathbf{0})$ in the AFI layer for different layer width w. For all temperatures, the ferromagnetic structure factor and the onset 

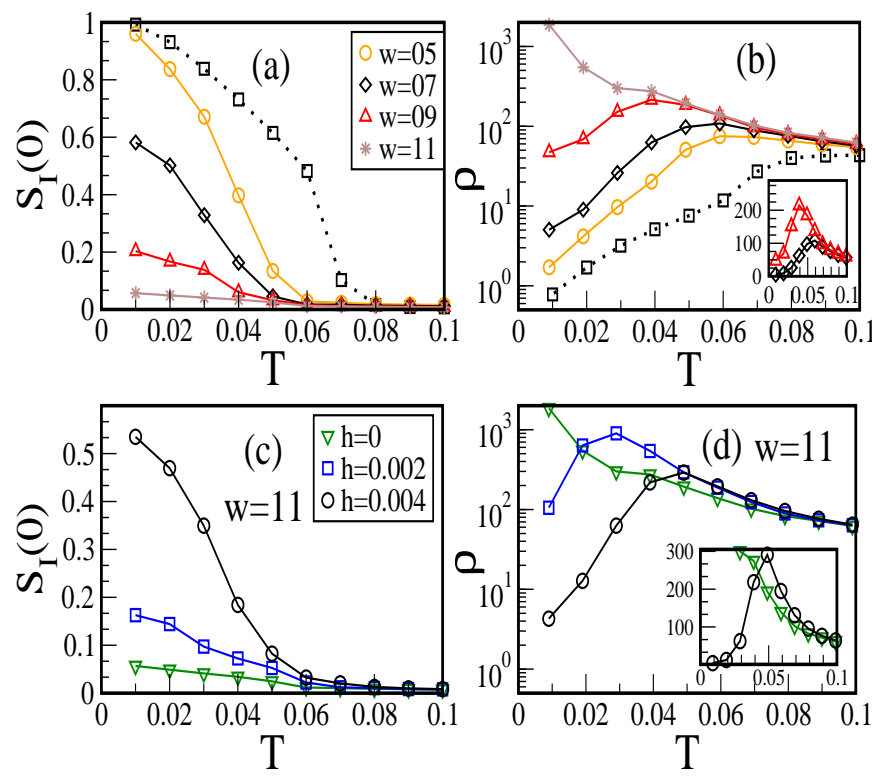

FIG. 8: Color online: Temperature dependence of (a) the ferromagnetic structure factor $S_{I}(\mathbf{0})$ and (b) the resistivity $\rho$ for different widths of the AFI layer. The dotted lines in (a) and (b) are for the bulk FM. (c) $S_{I}(\mathbf{0})$ and (d) resistivity variation with temperature for $\mathrm{w}=11$ in the presence of the external magnetic fields $h=0.002$ and 0.004 . Insets in (b) and (d) show the resistivity variation in the linear temperature scale.

temperature for ferromagnetism decrease with increasing w. The z component of the $t_{2 g}$ spins $\left\langle S_{z i}\right\rangle$ and the electron densities for each site in the superlattice are shown in Fig.1 (b) for $\mathrm{w}=11$. The two lines in the AFI layer nearest to the interface are aligned ferromagnetically to the $t_{2 g}$ spins on the FM side in accordance with the discussion in Section IV. The magnetic structure of the center lines is already similar to the bulk AFI phase, and the density profile reveals the emergence of local charge ordering patterns in the AFI layer. The superlattice for $\mathrm{w}$ $=11$ is therefore likely to be an insulator.

Fig.8(b) displays the temperature dependence of the longitudinal resistivity $\rho$ of the superlattice. $\rho$ for $\mathrm{w}=$ 11 steeply rises towards low temperatures as expected for an insulator. For $\mathrm{w} \leq 7$, the superlattice is ferromagnetic and metallic at low temperatures. For $\mathrm{w}=5$, the onset temperature for ferromagnetism in the AFI layer is near 0.05 , and simultaneously the resistivity start to decrease.

The low-temperature resistivity increases monotonously with the AFI layer width. An MIT occurs beyond a threshold width similar as in the experiments by $\mathrm{Li}$ et al ${ }^{30}$ who measured the variations in the resistivity in $\mathrm{LCMO} / \mathrm{PCMO}$ superlattices for different widths of the PCMO layer. In addition, the inset in Fig.8(b) shows a hump in the resistivity for $\mathrm{w}$ $=9$ and $\mathrm{w}=7$ at the ferromagnetic onset temperatures $\mathrm{T} \simeq 0.04$ and 0.05 , respectively, while there is no such hump in the bulk FM manganite (see the dashed line of Fig.8(b)). The humps in the resistivity curves increase and shift to lower temperatures with increasing AFI layer width (for $\mathrm{w} \leq 9$ ). This trend agrees well with the $\mathrm{LCMO} / \mathrm{PCMO}$ superlattice experiment ${ }^{30}$ with the exception of the experimentally observed hump in the bulk FM limit. The temperature dependence of $\rho$ near the MIT in bulk FM manganites is tied to the presence of intrinsic inhomogeneities and disorder ${ }^{59.60}$. Here we have not included any disorder $\underline{42}$ in the Hamiltonian; the MIT in the FM/AFI superlattice has a different origin. It is due to the induced onset of ferromagnetism in the AFI layer.

The natural next step is to explore the effect of an external magnetic field on the MIT in the superlattice. Fig.8(d) shows that the resistivity for $\mathrm{w}=11$ is lowered by the magnetic field at low temperatures. The connection between the resistivity decrease and the onset of ferromagnetism in the AFI layer is verified in Fig.8(c). The MIT for $h=0.004$ is at $T \simeq 0.05$; above this temperature, the resistivity is larger than the resistivity calculated for $\mathrm{h}=0$. The magnetoresistance, $[\rho(h)-\rho(0)] / \rho(0)$ therefore changes sign near the onset temperature for ferromagnetism.

At higher temperatures, the $\mathrm{t}_{2 g}$ spins are randomly oriented. We recall that in the limit $J_{H} \rightarrow \infty$ the $e_{g}$ electron spins are perfectly aligned along the local $t_{2 g}$ spin direction. So the current is equally carried by upspin and down-spin electrons. In the presence of a weak external magnetic field, the number of $e_{g}$ electrons is increased in the up-spin channel only in the FM layers and the tunneling current from the down-spin channel is decreased. The up-spin channel in the AFI layer remains unaltered and restricts the possible enhancement of the tunneling current from the up-spin channel. This results in an overall increase of the resistivity and a positive magnetoresistance.

At low temperatures in an external magnetic field the $\mathrm{t}_{2 g}$ spins in the AFI layer tend to align in the same direction as the $\mathrm{t}_{2 g}$ spins in the FM layers. So the tunneling current in the down-spin channel decreases but it increases for the up-spin channel. This decreases the overall resistivity of the superlattice. The corresponding crossover of the magnetoresistance from negative to positive was indeed reported in $\mathrm{La}_{0.7} \mathrm{Ce}_{0.3} \mathrm{MnO}_{3} / \mathrm{SrTiO}_{3}-$ $\mathrm{Nb}=61$ and $\mathrm{La}_{0.82} \mathrm{Ca}_{0.18} \mathrm{MnO}_{3} / \mathrm{SrTi}_{0.95} \mathrm{Nb}_{0.05} \mathrm{O}_{3} \frac{62}{6}$ heterojunctions.

The $\mathrm{z}$ component of the $\mathrm{t}_{2 g}$ spins and the electron densities for each site in the superlattice are shown in Fig.1(c) for $\mathrm{w}=11$ and $\mathrm{h}=0.004$. Ferromagnetic and AF structures coexist spatially separated in the AFI layer. The ferromagnetic phase in the upper panel is directly connected to the charge disordered regions in the lower panel. A magnetic field $\mathrm{h} \geq 0.007$ is required to tune the parent AFI into a ferromagnetic metal. Lower fields are instead required for the FM/AFI superlattices which are therefore candidates to design materials with large magnetoresistance. 

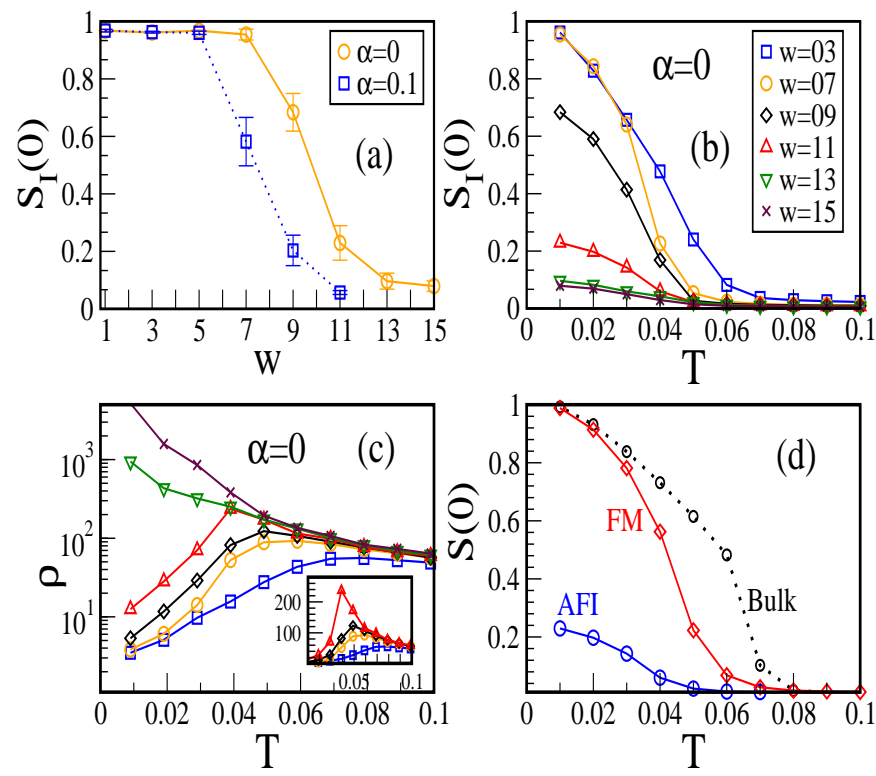

FIG. 9: Color online: For $\alpha=0$ (a) $S_{I}(\mathbf{0})$ for different widths of the AFI layers at $\mathrm{T}=0.01$. $S_{I}(\mathbf{0})$ for $\alpha=0.1$ is also replotted as the dotted line. Temperature dependence of (b) $S_{I}(\mathbf{0})$ and (c) the resistivity for different widths of the AFI layers. Legends in (b) and (c) are the same. Inset in (c) shows the temperature dependence of the resistivity in the linear scale. (d) The temperature dependence of ferromagnetic structure factor for the AFI layer, the FM layer for $\mathrm{w}=$ 11, and the bulk FM.

\section{RESULTS WITHOUT LRC INTERACTIONS}

In order to analyze the effect of the LRC interactions we examine the ferromagnetic structure factor $\mathrm{S}_{I}(\mathbf{0})$ for $\alpha=0$ in Fig.9(a). The critical width, beyond which $\mathrm{S}_{I}(\mathbf{0})$ starts to decrease, is $\mathrm{w}_{c}=7$. With the LRC potentials, $\alpha=0.1$, the critical width $\mathrm{w}_{c}=5$, as discussed in section $\mathrm{IV}$, is smaller due to the decrease in the average electron density in the AFI layer which is evident in Fig.7(a). All the discussions in the previous two sections remain qualitatively valid also for $\alpha=0$, but with a larger critical width $\mathrm{w}_{c}$.

In Fig.9(b), we plot the temperature dependence of $S_{I}(\mathbf{0})$ in the AFI layer for $\alpha=0$. Similar to the results in Fig.8(a), the onset temperature for ferromagnetism decreases with increasing w. Fig.9(c) shows the temperature dependence of the longitudinal resistivity of the superlattice. For $\mathrm{w}=13$ and $\mathrm{w}=15$, the low-temperature resistivity rises by orders of magnitude. The inset of Fig.9(c) zooms into the MIT at intermediate temperatures on a linear scale. The humps in $\rho(\mathrm{T})$ decrease and shift to higher temperatures for $\mathrm{w}<11$.

The onset temperature of ferromagnetism is higher in the FM layers than the AFI layers as becomes obvious from the temperature dependent structure factor in Fig.9(d). So there are two ferromagnetic transitions in the superlattice: the first at $\mathrm{T}_{c 1}$ for ferromagnetism in the FM layers and the second for the global ferromag-
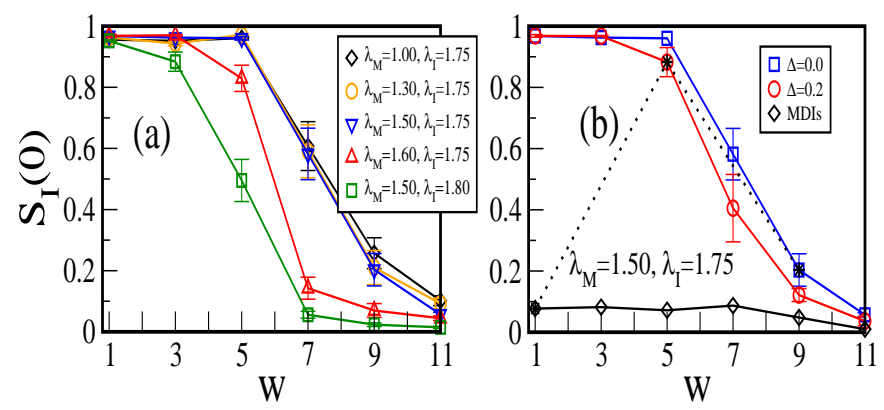

FIG. 10: Color online: $S_{I}(\mathbf{0})$ for different combinations of $\lambda_{M}$ and $\lambda_{I}$ values at $\mathrm{T}=0.01$. (b) $S_{I}(\mathbf{0})$ without $(\Delta=0)$ and with $(\Delta=0.2)$ quenched disorder, and with magnetically disordered interfaces (MDIs) (see text) for $\lambda_{M}=1.50$ and $\lambda_{I}=1.75$ at $\mathrm{T}=0.01$. The dotted line join the three points namely $\mathrm{w}=1$ (for MDIs), $\mathrm{w}=5$ (for $\Delta=0.2$ ), and $\mathrm{w}=9$ (for $\Delta=0$ ).

netism at $\mathrm{T}_{c 2}<\mathrm{T}_{c 1}$. The rise in the resistivity $\rho$ near the hump just below $\mathrm{T}_{c 1}$ is apparently due to the onset of ferromagnetism in the FM layers. The rise in $\rho$ is expected for the same reason as for positive magnetoresistance discussed in section $\mathrm{V}$. The downturn in $\rho$ results from the onset of global ferromagnetism in the superlattices at $\mathrm{T}_{c 2}$. These two transition temperatures are also observed in the LCMO/PCMO superlattices 30 . The temperature dependence of the ferromagnetic structure factor in the bulk FM state is included as the dotted line in Fig.9(d). The ferromagnetic onset temperature for the FM layers in the FM/AFI superlattices is lower than for the bulk.

\section{VARIATION OF $\lambda_{M}$ AND $\lambda_{I}$}

In this section we compare $S_{I}(\mathbf{0})$ for different combinations of electron-phonon couplings $\lambda_{M}$ and $\lambda_{I}$. In the previous sections $\lambda_{M}=1.5$ and $\lambda_{I}=1.75$ were chosen. If $\lambda_{I}$ is kept fixed and $\lambda_{M}$ varies between 1.0 and 1.6, the groundstate is ferromagnetic and metallic in the bulk limit at $n=0.6$. The induced magnetization in the AFI layer changes little for $\lambda_{M} \leq 1.5$ and for different widths w (see Fig.10(a)). For $\lambda_{M}=1.6$ however, $S_{I}(\mathbf{0})$ is reduced for $\mathrm{w} \geq 5$. The difference results from the decrease in the electron density in the FM layers nearest to the interface as becomes evident from the comparison with the bulk phase at $n=0.5 \underline{42}$. For $\lambda_{M} \leq 1.5$, the groundstate at $n=0.5$ is a ferromagnetic metal while it is a CE-type antiferromagnet for $\lambda_{M}=1.6$. For $\lambda_{M} \leq 1.5$, the magnetization in the interfacial FM layers is not altered, even if the electron density decreases towards $n=0.5$. But for $\lambda_{M}=1.60$, ferromagnetic order becomes unstable at the interfacial lines. The smaller magnetization at the interfacial FM layers decreases the induced magnetization in the AFI layer for $\mathrm{w} \geq 5$.

Fig.10(a) also indicates the reduced magnetization in the AFI layer for the larger electron-phonon coupling 
$\lambda_{I}=1.8$, for which the AFI layer recovers the AF, charge ordered state at a smaller width $\mathrm{w}$. It is therefore easier to induce a ferromagnetic moment in large-bandwidth (small $\lambda$ ) manganites. At $n=0.67$, the bandwidth of $\mathrm{PCMO}$ is the largest among those manganites for which an AF, charge ordered insulating phase is experimentally observed $\underline{16}$. In fact, in most of the experimental FM/AFI superlattices at $n=0.67$ the insulating manganite PCMO is used along with a variety of different FM manganites.

\section{DISORDER AT THE INTERFACE}

The size mismatch of $\mathrm{RE}$ and $\mathrm{AE}$ elements in the manganites $\mathrm{RE}_{1-x} \mathrm{AE}_{x} \mathrm{MnO}_{3}$ leads to tilting and distortions of the $\mathrm{MnO}_{6}$ octahedra and variations in the local electronic parameters. The tilting and distortions of $\mathrm{MnO}_{6}$ octahedra is generally known as A-type disorder. Here we have neglected the intrinsic A-type disorder in both the FM and the AFI manganites. Their interface is more prone to disorder due to chemical intermixing, lattice mismatch, and A-type disorder. We test the effect of quenched binary disorder in the terminating line of the FM layers at the interface by adding the potential disorder term by $\sum_{j} \epsilon_{j} n_{j}$ to the Hamiltonian. The sum over $\mathrm{j}$ is restricted to the terminating lines of the FM layers and $\epsilon_{j}$ is the quenched disorder potential with $\bar{\epsilon}_{j}=0$ and values $\pm \Delta$.

In Fig.10(b), we show the induced magnetization in the AFI layer for $\Delta=0.2$ and different widths w, which apparently changes little between $\Delta=0$ and $\Delta=0.2$. All the discussions in the previous sections remain qualitatively unchanged for moderate disorder $\Delta=0.2$. The magnetization of the interfacial lines of the FM layers, where $\Delta$ is included, is nevertheless likely to decrease for larger $\Delta$. Ultimately the magnetization in those interfacial lines will be quenched due to spin disorder. In order to see the effect of the magnetically disordered interfaces on the AFI layer we have fixed randomly oriented spins in the interfacial lines throughout the Monte Carlo simulations. Indeed, in this extreme case, the induced magnetization in the AFI layer is very small (see Fig.10(b)), irrespective of the AFI layer width. The magnetization of the FM layer at the interface is therefore crucial to induce the ferromagnetic moments in the AFI layer.

In the experiments it is apparently not clear whether or not there are magnetically disordered interfaces on the FM sides in the FM/AFI superlattices 34 . Thin AFI layers are likely to be more susceptible to disorder due to strain effects. The disorder strength $\Delta$ at the interface will therefore increase with decreasing the AFI layer width; the width dependence of the disorder strength is however hard to quantify. If we combine the results in Fig.10(b) such that disorder strength is maximum for smaller AFI layers width and decreases thereafter for larger $\mathrm{w}$ (see the dotted line) then the magnetic moment in the AFI layer behaves non-monotonically which agrees qualitatively with the LSMO/PCMO superlattice experiment ${ }^{34}$. But more theoretical work is needed to understand this non-monotonic behaviour.

\section{CONCLUSIONS}

We have investigated the magnetic and electronic properties of manganite superlattices at the specific electron density $n=0.6$ using a two-orbital double-exchange model including super-exchange interactions, JT distortions, and LRC interactions in 2D. At the interface electrons are transferred from the the FM to the AFI manganites. Friedel-like density oscillations are observed in both the FM and the AFI layers. Due to the charge transfer and the induced magnetization the smaller width $\mathrm{AF}$ insulator sandwiched between FM manganites turns into a ferromagnetic metal at low temperatures. The induced magnetization in the AFI layer decreases beyond a critical width and away from the interface the AFI layer gradually returns to the bulk AFI state. This results in an MIT in the FM/AFI superlattice driven by the AFI layer width. Only weak external magnetic field is required to tune the insulating into a metallic state. The sign of the magnetoresistance changes at higher temperatures. A remarkable result is that colossal magnetoresistance in manganite superlattices is achieved at lower magnetic fields as in the bulk phases of the constituent materials. For the range of AFI layer widths, at which FM/AFI superlattices show a metallic behavior at low temperatures, a hump in the temperature dependence of the resistivity $\rho$ is observed. The hump in $\rho$ is due to the presence of two ferromagnetic transition temperatures in the superlattice. The height of the hump decreases and shifts to higher temperatures with decreasing AFI layer width.

In summary, the width of the AFI layers controls the magnitude of the magnetoresistance and the MIT in the FM/AFI superlattices. Our 2D model Hamiltonian calculations provide a basis for explaining the MIT in manganite superlattices. The non-monotonic behaviour of the induced ferromagnetic moment in the AFI layer is traced to the effects of magnetic disorder at the interface.

\section{ACKNOWLEDGEMENT}

This work was supported by the Deutsche Forschungsgemeinschaft through TRR80. We acknowledge helpful discussions with Sanjeev Kumar. 
1 E. Dagotto, Science 309, 257 (2005).

2 Y. Tokura, Rep. Prog. Phys. 69, 797 (2006).

${ }^{3}$ H. Y. Hwang, Y. Iwasa, M. Kawasaki, B. Keimer, N. Nagaosa, and Y. Tokura, Nature Mater. 11, 103 (2012).

4 J. Heber, Nature 459, 28 (2009).

${ }^{5}$ P. Zubko, S. Gariglio, M. Gabay, P. Ghosez, and J. M. Triscone, Ann. Rev. of Condens. Matter Phys. 2, 141 (2011).

6 A. Ohtomo and H. Y. Hwang, Nature 427, 423 (2004).

7 N. Reyren, S. Thiel, A. D. Caviglia, L. Fitting-Kourkoutis, G. Hammerl, C. Richter, C. W. Schneider, T. Kopp, A. S. Ruetschi, D. Jaccard, M. Gabay, D. A. Muller, J. M. Triscone, and J. Mannhart, Science 317, 1196 (2007).

8 A. Brinkman, M. Huijben, M. van Zalk, J. Huijben, U. Zeitler, J. C. Maan, W. G. van der Wiel, G. Rijnders, D. H. A. Blank, and H. Hilgenkamp, Nature Mater. 6, 493 (2007).

9 D. A. Dikin, M. Mehta, C. W. Bark, C. M. Folkman, C. B. Eom, and V. Chandrasekhar, Phys. Rev. Lett. 107, 056802 (2011).

10 J. Mannhart and D. G. Schlom, Science 327, 1607 (2010).

11 Colossal Magnetoresistive Oxides, edited by Y. Tokura (Gordon and Breach, New York, 2000).

12 Colossal Magnetoresistive Manganites, edited by T. Chatterji (Springer-Verlag, Berlin, 2004).

13 E. Dagotto, Nanoscale Phase Separation and Colossal Magnetoresistance: The Physics of Manganites and Related Compounds, Springer Series in Solid State Sciences, Vol. 136 (2005).

14 S. Jin, T. H. Tiefel, M. McCormack, R.A. Fastnacht, R. Ramesh, and L.H. Chen, Science 264, 413 (1994).

15 E. Dagotto, New J. Phys. 7, 67 (2005).

16 R. Kajimoto, H. Yoshizawa, Y. Tomioka, and Y. Tokura, Phys. Rev. B 66, 180402 (2002).

17 E. Wollan and W. Koehler, Phys. Rev. 100, 545 (1955).

18 J. Hemberger, A. Krimmel, T. Kurz, H. A. Krug von Nidda, V. Yu. Ivanov, A. A. Mukhin, A. M. Balbashov, and A. Loidl, Phys. Rev. B 66, 094410 (2002).

19 O. Chmaissem, B. Dabrowski, S. Kolesnik, J. Mais, J. D. Jorgensen, and S. Short, Phys. Rev. B 67, 094431 (2003).

20 A. Bhattacharya, S. J. May, S. G. E. te Velthuis, M. Warusawithana, X. Zhai, B. Jiang, J. M. Zuo, M. R. Fitzsimmons, S. D. Bader, and J. N. Eckstein, Phys. Rev. Lett. 100, 257203 (2008)

21 C. Adamo, X. Ke, P. Schiffer, A. Soukiassian, M. Warusawithana, L. Maritato, and D. G. Schlom, Appl. Phys. Lett. 92, 112508 (2008).

22 S. J. May, P. J. Ryan, J. L. Robertson, J.-W. Kim, T. S. Santos, E. Karapetrova, J. L. Zarestky, X. Zhai, S. G. E. te Velthuis, J. N. Eckstein, S. D. Bader, and A. Bhattacharya, Nature Mater. 8, 892 (2009).

23 C. Lin and A. J. Millis, Phys. Rev. B 78, 184405 (2008).

24 B. R. K. Nanda and S. Satpathy, Phys. Rev. B 78, 054427 (2008).

25 R. Yu, S. Yunoki, S. Dong, and E. Dagotto, Phys. Rev. B 80, 125115 (2009).

26 L. Brey, Phys. Rev. B 75, 104423 (2007).

27 S. Dong, R. Yu, S. Yunoki, G. Alvarez, J. M. Liu, and E. Dagotto, Phys. Rev. B 78, 201102(R) (2008).

28 R. Cheng, K. Li, S. Wang, Z. Chen, C. Xiong, X. Xu, and Y. Zhang, Appl. Phys. Lett. 72, 2475 (1998).
29 I. N. Krivorotov, K. R. Nikolaev, A. Yu. Dobin, A. M. Goldman, and E. Dan Dahlberg, Phys. Rev. Lett. 86, 5779 (2001).

30 H. Li, J. R. Sun, and H. K. Wong, Appl. Phys. Lett. 80, 628 (2002).

31 M. Jo, M. G. Blamire, D. Ozkaya, and A. K. Petford-Long, J. Phys.: Condens. Matter 15, 5243 (2003).

32 M. N. Baibich, J. M. Broto, A. Fert, F. Nguyen Van Dau, F. Petroff, P. Etienne, G. Creuzet, A. Friederich, and J. Chazelas, Phys. Rev. Lett. 61, 2472 (1988).

33 G. Binasch, P. Grünberg, F. Saurenbach, and W. Zinn, Phys. Rev. B 39, 4828 (1989).

34 D. Niebieskikwiat, M. B. Salomon, L. E. Hueso, N. D. Mathur, and J. A. Borchers, Phys. Rev. Lett. 99, 247207 (2007).

35 G. Lian, Z. Wang, J. Gao, J. Kang, M. Li, and G. Xiong, J. Phys. D 32, 90 (1999).

36 S. Mukhopadhyay and I. Das, Europhys. Lett. 83, 27003 (2008).

37 D. Niebieskikwiat, L. E. Hueso, N. D. Mathur, and M. B. Salomon, Appl. Phys. Lett. 93, 123120 (2008).

38 J. Salafranca, M. J. Calderon, and L. Brey, Phys. Rev. B 77, 014441 (2008).

39 S. Yunoki, T. Hotta, and E. Dagotto, Phys. Rev. Lett. 84, 3714 (2000).

40 E. Dagotto, T. Hotta, and A. Moreo, Phys. Rep. 344, 1 (2001).

41 S. Kumar, A. P. Kampf, and P. Majumdar, Phys. Rev. Lett. 97, 176403 (2006).

42 K. Pradhan, A. Mukherjee, and P. Majumdar, Phys. Rev. Lett. 99, 147206 (2007).

43 E. Dagotto, S. Yunoki, A. L. Malvezzi, A. Moreo, J. Hu, S. Capponi, D. Poilblanc, and N. Furukawa, Phys. Rev. B 58, 6414 (1998).

44 A. C. Green, Phys. Rev. B 63, 205110 (2001).

45 Z. Popovic and S. Satpathy, Phys. Rev. Lett. 88, 197201 (2002).

46 Y. Okimoto, T. Katsufuji, T. Ishikawa, A. Urushibara, T. Arima, and Y. Tokura, Phys. Rev. Lett. 75, 109 (1995).

47 K. Pradhan, A. Mukherjee, and P. Majumdar, Europhys. Lett. 84, 37007 (2008).

48 S. Okamoto and A. J. Millis, Phys. Rev. B 70, 075101 (2004)

49 S. Yunoki, A. Moreo, E. Dagotto, S. Okamoto, S. S. Kancharla, and A. Fujimori, Phys. Rev. B 76, 064532 (2007).

50 Surfaces and interfaces of solids, H. Luth (Springer-Verlag, Berlin; New York, 1993).

51 P. Lunkenheimer, V. Bobnar, A. V. Pronin, A. I. Ritus, A. A. Volkov, and A. Loidl, Phys. Rev. B 66, 052105 (2002).

52 J. L. Cohn, M. Peterca, and J. J. Neumeier, Phys. Rev. B 70, 214433 (2004).

53 B. Korenblum and E. I. Rashba, Phys. Rev. Lett. 89, 096803 (2002).

54 T. Oka and N. Nagaosa, Phys. Rev. Lett. 95, 266403 (2005).

55 S. Kumar and P. Majumdar, Eur. Phys. J. B 50, 571 (2006).

56 T. G. Perring, G. Aeppli, Y. Moritomo, and Y. Tokura, Phys. Rev. Lett. 78, 3197 (1997).

57 G. D. Mahan, Quantum Many Particle Physics (Plenum Press, New York, 1990). 
58 S. Kumar and P. Majumdar, Eur. Phys. J. B 46, 237 (2005).

59 J. Burgy, M. Mayr, V. Martin-Mayor, A. Moreo, and E. Dagotto, Phys. Rev. Lett. 87, 277202 (2001).

60 Y. Motome, N. Furukawa, and N. Nagaosa, Phys. Rev. Lett. 91, 167204 (2003).
61 Z. G. Sheng, W. H. Song, Y. P. Sun, J. R. Sun, and B. G. Shen, Appl. Phys. Lett. 87, 032501 (2005).

62 T. F. Zhou, G. Li, N. Y. Wang, B. M. Wang, X. G. Li, and Y. Chen, Appl. Phys. Lett. 88, 232508 (2006). 